\title{
CENTRAL LIMIT THEOREMS FOR FUNCTIONALS OF STATIONARY GERM-GRAIN MODELS
}

\author{
URSA PANTLE, ${ }^{*}$ \\ VOLKER SCHMIDT $* * *$ AND \\ EVGUENI SPODAREV, ${ }^{*}$ Universität Ulm
}

\begin{abstract}
Conditions are derived for the asymptotic normality of a general class of vector-valued functionals of stationary Boolean models in the $d$-dimensional Euclidean space, where a Lindeberg-type central limit theorem for $m$-dependent random fields, $m \in \mathbb{N}$, is applied. These functionals can be used to construct joint estimators for the vector of specific intrinsic volumes of the underlying Boolean model. Extensions to functionals of more general germ-grain models satisfying some mixing and integrability conditions are also discussed.

Keywords: Random closed set; Boolean model; stationary random field; $m$-dependent random field; valuation; asymptotic normality; $\beta$-mixing; specific intrinsic volume; Euler number

2000 Mathematics Subject Classification: Primary 60D05
\end{abstract}

Secondary 60F05; 62M40

\section{Introduction}

Consider a stationary random closed set $\Xi \subset \mathbb{R}^{d}$ such that $\Xi \cap K$ belongs to the convex ring $\mathcal{R}$ with probability 1 for any convex and compact test set $K \subset \mathbb{R}^{d}$. Assume that $\Xi$ can be (indirectly) observed within a bounded observation window $W \subset \mathbb{R}^{d}$. Suppose that this indirect observation is made by measuring some 'local' geometric features,

$$
Y(x)=f((\Xi-x) \cap K), \quad x \in W \ominus \check{K},
$$

of $\Xi$ within a small scanning window $K \subset \mathbb{R}^{d}$, where ' $\ominus$ ' denotes Minkowski difference, $\check{K}$ is the reflection of $K$, and $f: \mathcal{R} \rightarrow \mathbb{R}$ is some real-valued functional possessing the properties of a valuation (see, e.g. [13, p. 184]). If $f$ is invariant with respect to translations, then $Y(x)=f(\Xi \cap(K+x))$ holds, where $K+x$ can be interpreted as local neighborhood of the measurement point $x$. A natural unbiased estimator for the mean $\mu=\mathrm{E}(Y(x))$ of the stationary random field $Y=\left\{Y(x), x \in \mathbb{R}^{d}\right\}$ is the weighted average

$$
\hat{\mu}=\int_{W} Y(x) G(W, x) \mathrm{d} x,
$$

where $G(W, x)$ is a weighting kernel that integrates to 1 over $W$ and vanishes for those $x$ for which $Y(x)$ is not observable.

Received 21 March 2005; revision received 19 September 2005.

* Postal address: Abteilung Stochastik, Universität Ulm, Helmholtzstr. 18, D-89069 Ulm, Germany.

** Email address: volker.schmidt@uni-ulm.de

Presented at the ICMS Workshop on Spatial Stochastic Modelling with Applications to Communications Networks (Edinburgh, June 2004). 
The question to be answered is, what asymptotic properties does the estimator $\hat{\mu}$ have for an unboundedly increasing sequence of observation windows $W_{n} \uparrow \mathbb{R}^{d}, n \in \mathbb{N}$ ? It is well known from the general theory of stationary random fields (see, e.g. Section 1.7 of [8, pp. 35-42]) that the estimator given in (1.2), properly normalized, is asymptotically normally distributed under the assumption that $\mathrm{E}\left(|Y(x)|^{2+\delta}\right)<\infty$ for some $\delta>0$ and if additional Rosenblatt-type mixing conditions on $Y$ are satisfied. Roughly speaking, these conditions ensure that various mixing rates of $Y$ expressing the dependence between $Y(x)$ and $Y(x+t)$ decrease in order of $|t|^{-d-\varepsilon}$, as $|t| \rightarrow \infty$ and for some $\varepsilon>0$. Notice that these assumptions are dictated by the sectioning technique of Bernstein and the classical Lyapunov-form central limit theorem used in the proofs.

However, in the context of random fields $Y$ as defined in (1.1) and generated by random closed sets of the form $\Xi=\bigcup_{i=1}^{\infty}\left(M_{i}+X_{i}\right)$, where $\left\{X_{i}\right\}$ is a point process of 'germs' and $\left\{M_{i}\right\}$ is a sequence of random compact 'grains', a mixing condition on $\left\{X_{i}\right\}$ and an integrability condition on $\left\{M_{i}\right\}$ can be used to show the asymptotic normality of the estimator $\hat{\mu}$ given in (1.2). In particular, if $\left\{X_{i}\right\}$ is a Poisson process or a 'Poisson-like' point process with finite range of correlation, a Lindeberg-type central limit theorem developed in [4] for so-called $m$-dependent random fields, $m \in \mathbb{N}$, is applicable.

We emphasize that this technique can be used to prove the asymptotic normality of $\hat{\mu}$ for any conditionally bounded valuation $f$. Related results for another general class of functionals of germ-grain models have been derived in [7]. Furthermore, there exist various results of this sort for particular functionals $f$, such as the empirical volume fraction, boundary length, and convexity number; see, e.g. [1], [5], [9], and the references in [10, pp. 30-43].

The paper is organized as follows. Section 2 contains preliminary results. In Section 2.1, we recall some basic notions from stochastic geometry, such as random closed sets, germgrain models, and, in particular, the Boolean model. Then, in Section 2.2, a quite general class of functionals of stationary random fields is introduced and an upper bound is derived for the moments of stationary random fields associated with these functionals. In Section 2.3, conditions for the mean-square consistency of the mean-value estimator $\hat{\mu}$ are given. Some examples of valuations are discussed in Section 3. The corresponding random fields can be used to construct joint estimators for the vector of specific intrinsic volumes of stationary random sets; see [11] and [14]. In Section 4, we consider a Boolean model $\Xi=\bigcup_{i=1}^{\infty}\left(M_{i}+X_{i}\right)$ with convex and compact grains. In particular, we show in Section 4.1 that the covariance function $\operatorname{cov}_{Y}(x)$ admits an integrable upper bound provided that

$$
\mathrm{E}\left(\left|M_{i} \oplus \check{K}\right|^{2}\right)<\infty
$$

where ' $\oplus$ ' denotes Minkowski sum and $|\cdot|$ is the $d$-dimensional Lebesgue measure. This bound depends on the distribution of the grains $M_{i}$; the dependence is monotone with respect to inclusion. Using a truncation technique and the Lindeberg-type central limit theorem for $m$-dependent random fields, we show in Sections 4.2 and 4.3 that the weighted average $\hat{\mu}$ of $Y$ over $W$ is asymptotically normally distributed for any unboundedly increasing sequence of observation windows $W_{n} \uparrow \mathbb{R}^{d}$ that satisfies certain additional regularity conditions. Using the well-known Cramér-Wold device, this result can be easily extended to a multidimensional setting.

Conditions for the asymptotic normality of the estimator $\hat{\mu}$ for more general germ-grain models are discussed in Section 5. Proceeding as in [7], a central limit theorem for $\beta$-mixing random fields given in [6] is applied, together with an upper bound for the $\beta$-mixing coefficient of random measures associated with the germ-grain models. For this theorem, a stronger 
integrability condition is needed, namely $\mathrm{E}\left(D^{2 d\left(1+\delta^{\prime}\right)}\left(M_{i}\right)\right)<\infty$ for some $\delta^{\prime}>0$, where $D\left(M_{i}\right)=\sup \left\{|x|, x \in M_{i}\right\}$ is the 'radius' of the grains. Notice that condition (1.3) is fulfilled if $\mathrm{E}\left(D^{2 d}\left(M_{i}\right)\right)<\infty$.

\section{Mean-value estimators for stationary random fields}

We first recall some basic notions from stochastic geometry that will be used in the paper. Further details can be found in, e.g. [13] and [15]. In the second part of this section, we consider a class of unbiased and consistent estimators for the mean value of certain stationary random fields.

\subsection{Germ-grain models}

Let $d \geq 2$ be an arbitrary, fixed integer. For any two sets $B, B^{\prime} \subset \mathbb{R}^{d}$, let $B \oplus B^{\prime}=$ $\left\{x+y, x \in B, y \in B^{\prime}\right\}$ be the Minkowski sum of $B$ and $B^{\prime}$ and write $B+x=B \oplus\{x\}$ for the translation of $B$ by the vector $x \in \mathbb{R}^{d}$. Furthermore, consider the reflection $\breve{B}=\{-x, x \in B\}$ of $B$ at the origin and denote the Minkowski difference of $B$ and $B^{\prime}$ by $B \ominus B^{\prime}=\left\{x: \check{B}^{\prime}+x \subseteq B\right\}$.

Let $\mathcal{B}\left(\mathbb{R}^{d}\right)$ be the $\sigma$-algebra of Borel sets in $\mathbb{R}^{d}$ and let $\mathcal{B}_{0}\left(\mathbb{R}^{d}\right) \subset \mathcal{B}\left(\mathbb{R}^{d}\right)$ be the family of all bounded Borel sets. Furthermore, let $\mathcal{F} \subset \mathscr{B}\left(\mathbb{R}^{d}\right)$ denote the family of all closed sets and $\mathcal{K} \subset \mathcal{F}$ the family of all convex bodies, i.e. convex and compact sets in $\mathbb{R}^{d}$. For the convex ring we shall write $\mathcal{R}$. It is the family of all finite unions of sets in $\mathcal{K}$, which are sometimes also called polyconvex sets. The extended convex ring $\&$ is the family of Borel sets $B \in \mathcal{B}\left(\mathbb{R}^{d}\right)$ such that $B \cap K \in \mathcal{R}$ holds for any convex body $K \in \mathcal{K}$. A random closed set (RACS) $\Xi$ in $\mathbb{R}^{d}$ is an $\left(\mathcal{A}, \sigma_{\mathcal{F}}\right)$-measurable mapping from some probability space $(\Omega, \mathcal{A}, \mathrm{P})$ into $\mathscr{F}$ equipped with the $\sigma$-algebra $\sigma_{\mathcal{F}}$, which is generated by the events $\{F \in \mathcal{F}, F \cap K \neq \varnothing\}, K \in \mathscr{F}$, with $K$ compact.

We say that $\Xi$ is stationary if the distribution of the translated RACS $\Xi+x$ is equal to the distribution of $\Xi$ for any $x \in \mathbb{R}^{d}$. In the following, we consider stationary RACSs $\Xi$ with realizations from the extended convex ring \&, i.e. with $\Xi \cap K \in \mathcal{R}$ almost surely for any $K \in \mathcal{K}$. The RACS $\Xi$ is said to be an (independently marked) germ-grain model if it can be represented in the form

$$
\Xi=\bigcup_{i=1}^{\infty}\left(M_{i}+X_{i}\right),
$$

where the so-called germs $X_{i}$ form a simple point process $X=\left\{X_{i}\right\}$ in $\mathbb{R}^{d}$ and the sequence $M=\left\{M_{i}\right\}$ of grains $M_{i}$ is independent of $\left\{X_{i}\right\}$ and consists of independent copies of a nonempty compact RACS $M_{0}$. Notice that the infinite union of RACSs $M_{i}+X_{i}$ on the right-hand side of (2.1) is almost surely closed and different from $\mathbb{R}^{d}$ if the point process $X$ is stationary with finite intensity $\lambda$ and if

$$
\mathrm{E}\left(\left|M_{0} \oplus \check{K}\right|\right)<\infty
$$

for each $K \in \mathcal{K}$. This condition holds, for instance, if $\mathrm{E}\left(D^{d}\left(M_{0}\right)\right)<\infty$, where $D(B)=$ $\sup \{|x|, x \in B\}$ denotes the radius (or norm) of a Borel set $B \in \mathscr{B}\left(\mathbb{R}^{d}\right)$ and $|x|$ is the length of the vector $x \in \mathbb{R}^{d}$. Condition (2.2) and the stationarity of $X$ imply that only finitely many translated grains $M_{i}+X_{i}$ have a nonempty intersection $\left(M_{i}+X_{i}\right) \cap K$ with any fixed convex body $K \in \mathcal{K}$. In other words, the random variable

$$
N(\Xi \cap K)=\operatorname{card}\left\{i:\left(M_{i}+X_{i}\right) \cap K \neq \varnothing\right\}
$$

is finite with probability 1 for each $K \in \mathcal{K}$, where $\operatorname{card}(B)$ denotes the cardinality of the set $B$. Let $g_{N(\Xi \cap K)}(s)=\mathrm{E}\left(s^{N(\Xi \cap K)}\right), s \in \mathbb{R}$, be the generating function of $N(\Xi \cap K)$. 
If the point process $\left\{X_{i}\right\}$ of germs is a stationary Poisson process, then the stationary RACS $\Xi$ defined by formula (2.1) is called a Boolean model. For Boolean models, it is not difficult to show that the random variable $N(\Xi \cap K)$ is Poisson distributed with parameter $\lambda \mathrm{E}\left(\left|M_{0} \oplus \check{K}\right|\right)$; see, e.g. Section 4.1 of [3, p. 201]. Thus, in this case, the generating function $g_{N(\Xi \cap K)}$ is given by

$$
g_{N(\Xi \cap K)}(s)=\mathrm{e}^{(s-1) \lambda \mathrm{E}\left(\left|M_{0} \oplus \check{K}\right|\right)}, \quad s \in \mathbb{R} .
$$

This means in particular that $g_{N(\Xi \cap K)}(s)<\infty$ for any $s \in \mathbb{R}$ if $(2.2)$ is satisfied. Furthermore, a Boolean model $\Xi$ with nonempty polyconvex grains $M_{i}$ can be represented as the union set of a Poisson (particle) process $\tilde{M}=\left\{\tilde{M}_{i}\right\}$ on $\mathcal{R}$, where $\tilde{M}_{i}=M_{i}+X_{i}$; in other words, we have $\Xi=\bigcup_{i=1}^{\infty} \tilde{M}_{i}$ (see, e.g. [13, Section 4.4, p. 151]).

\subsection{Random fields associated with germ-grain models}

Let the functional $f: \mathcal{R} \rightarrow \mathbb{R}$ be a valuation on the convex ring $\mathcal{R}$. This means that $f(\varnothing)=0$ and that $f$ is measurable and additive, i.e.

$$
f\left(K_{1} \cup K_{2}\right)=f\left(K_{1}\right)+f\left(K_{2}\right)-f\left(K_{1} \cap K_{2}\right)
$$

for any $K_{1}, K_{2} \in \mathcal{R}$. Regarding the value $f\left(K_{1} \cup \cdots \cup K_{k}\right)$ for the union of $k \geq 2$ sets $K_{1}, \ldots, K_{k}$ from $\mathcal{R}$, the general inclusion-exclusion formula

$$
f\left(K_{1} \cup \cdots \cup K_{k}\right)=\sum_{i=1}^{k}(-1)^{i-1} \sum_{j_{1}<\cdots<j_{i}} f\left(K_{j_{1}} \cap \cdots \cap K_{j_{i}}\right)
$$

easily follows from the additivity of $f$. Furthermore, we assume that $f$ is conditionally bounded on $\mathcal{K}$, that is, for any pair $K, K^{\prime} \in \mathcal{K}$ with $K^{\prime} \subseteq K$, the inequality

$$
\left|f\left(K^{\prime}\right)\right| \leq c(K)
$$

holds for some finite bound $c(K)$. For any fixed convex body $K \in \mathcal{K}$ and for any RACS $\Xi$, consider the random field $Y=\left\{Y(x), x \in \mathbb{R}^{d}\right\}$ given by

$$
Y(x)=f((\Xi-x) \cap K), \quad x \in \mathbb{R}^{d} .
$$

If $\Xi$ is stationary then the random field $Y$ is stationary, i.e. its finite-dimensional distributions are invariant with respect to translations. In particular, we have $Y(x) \stackrel{\mathrm{D}}{=} Y(o)$ for any $x \in \mathbb{R}^{d}$, where ' $\stackrel{\text { D, }}{=}$ denotes equality in distribution and $o \in \mathbb{R}^{d}$ is the origin. Throughout this paper, we assume that the field $Y$ given by (2.5) is of second order, which means that

$$
\mathrm{E}\left(Y^{2}(x)\right)<\infty, \quad x \in \mathbb{R}^{d} .
$$

This condition implies that the covariance $\operatorname{cov}_{Y}(x)=\operatorname{cov}(Y(o), Y(x))$ is well defined for any $x \in \mathbb{R}^{d}$. Notice that a sufficient condition for the existence of the second moment of $Y$ can be provided in terms of the generating function $g_{N(\Xi \cap K)}(s)$ of the random variable $N(\Xi \cap K)$ defined in (2.3).

Lemma 2.1. Let $\Xi$ be a germ-grain model with $M_{0} \in \mathcal{R}$ such that the minimal number of convex components of $M_{0}$ is bounded by some constant $n_{0}<\infty$. Then

$$
\mathrm{E}\left(\left|Y^{p}(x)\right|\right) \leq c^{p}(K) g_{N(\Xi \cap K)}\left(2^{n_{0} p}\right)
$$

for any $p>0$ and $x \in \mathbb{R}^{d}$, where $c(K)$ is an upper bound for $\left|f\left(K^{\prime}\right)\right|$ for all $K^{\prime} \in \mathcal{K}$ with $K^{\prime} \subseteq K$. 
Proof. We prove the assertion only for the special case $n_{0}=1$, i.e. we assume that $M_{0} \in \mathcal{K}$. For any integer $m \geq 0$, we let $I_{m}(x)=\{N((\Xi-x) \cap K)=m\}$ and $p_{N(\Xi \cap K)}(m)=\mathrm{P}\left(I_{m}(o)\right)$. Then, using the properties of valuations, we obtain

$$
\begin{aligned}
\mathrm{E}\left|Y^{p}(x)\right| & =\sum_{m=1}^{\infty} \mathrm{E}\left(\left|f\left(\bigcup_{i=1}^{m}\left(M_{i}+X_{i}-x\right) \cap K\right)\right|^{p} \mid I_{m}(x)\right) p_{N(\Xi \cap K)}(m) \\
& =\sum_{m=1}^{\infty} \mathrm{E}\left(\mid \sum_{k=1}^{m}(-1)^{k-1} \sum_{i_{1}<\cdots<i_{k}} f\left(\left(M_{i_{1}}+X_{i_{1}}-x\right) \cap \cdots\right.\right. \\
& \left.\left.\left.\leq M_{i_{k}}+X_{i_{k}}-x\right) \cap K\right)\left.\right|^{p} \mid I_{m}(x)\right) p_{N(\Xi \cap K)}(m) \\
& \sum_{m=0}^{\infty}\left(\sum_{k=0}^{m}\left(\begin{array}{c}
m \\
k
\end{array}\right) c(K)\right)^{p} p_{N(\Xi \cap K)}(m) \\
& =c^{p}(K) \sum_{m=0}^{\infty} 2^{m p} p_{N(\Xi \cap K)}(m) \\
& =c^{p}(K) g_{N(\Xi \cap K)}\left(2^{p}\right),
\end{aligned}
$$

where the inequality is due to the conditional boundedness of $f$. The proof of the general case is similar and, therefore, omitted.

\subsection{Unbiased and consistent estimation of the mean}

Consider an unboundedly increasing sequence $\left\{W_{n}\right\}$ of bounded Borel sets $W_{n} \subset \mathbb{R}^{d}$ with

$$
\lim _{n \rightarrow \infty}\left|W_{n}\right|=\infty \quad \text { and } \quad \lim _{n \rightarrow \infty} \frac{\left|\partial W_{n} \oplus B_{r}(o)\right|}{\left|W_{n}\right|}=0 \quad \text { for any } r>0 .
$$

Here, $B_{r}(x)=\left\{y \in \mathbb{R}^{d}:|y-x| \leq r\right\}$ is the closed ball in $\mathbb{R}^{d}$ centered at $x \in \mathbb{R}^{d}$ with radius $r>0$, and $\partial B$ is the boundary of a Borel set $B$. Notice that (2.6) implies

$$
\lim _{n \rightarrow \infty} \frac{\left|W_{n} \oplus B_{r}(o)\right|}{\left|W_{n}\right|}=\lim _{n \rightarrow \infty} \frac{\left|W_{n} \ominus B_{r}(o)\right|}{\left|W_{n}\right|}=1 \quad \text { for any } r>0 .
$$

Thus, without loss of generality, we can assume that $\left|W_{n} \ominus \check{K}\right|>0$ for each $n \geq 1$. Furthermore, let $G: \mathcal{B}_{0}\left(\mathbb{R}^{d}\right) \times \mathbb{R}^{d} \rightarrow[0, \infty)$ be some nonnegative function Borel measurable in the second component such that, for each $n \geq 1$,

$$
G\left(W_{n}, x\right)=0 \quad \text { if } x \in \mathbb{R}^{d} \backslash\left(W_{n} \ominus \check{K}\right) \quad \text { and } \quad \int_{W_{n}} G\left(W_{n}, x\right) \mathrm{d} x=1 .
$$

Now assume that the RACS $\Xi$ is stationary. It then follows from Fubini's theorem that

$$
\hat{\mu}_{n}=\int_{W_{n}} Y(x) G\left(W_{n}, x\right) \mathrm{d} x
$$

is an unbiased estimator for the expectation $\mu=\mathrm{E}(Y(o))$, where $Y(x)$ is given by (2.5). Moreover, the estimation variance $\operatorname{var}\left(\hat{\mu}_{n}\right)$ can be determined as follows. 
Lemma 2.2. For any $n \geq 1$,

$$
\operatorname{var}\left(\hat{\mu}_{n}\right)=\int_{\mathbb{R}^{d}} \operatorname{cov}_{Y}(x) R_{W_{n}}(x) \mathrm{d} x,
$$

where $R_{W_{n}}(x)=\int_{\mathbb{R}^{d}} G\left(W_{n}, y\right) G\left(W_{n}, x+y\right) \mathrm{d} y$.

Proof. We have

$$
\begin{aligned}
\operatorname{var}\left(\hat{\mu}_{n}\right) & =\mathrm{E}\left(\int_{W_{n}}(Y(u)-\mu) G\left(W_{n}, u\right) \mathrm{d} u \int_{W_{n}}(Y(v)-\mu) G\left(W_{n}, v\right) \mathrm{d} v\right) \\
& =\int_{W_{n}} \int_{W_{n}} \mathrm{E}((Y(o)-\mu)(Y(v-u)-\mu)) G\left(W_{n}, u\right) G\left(W_{n}, v\right) \mathrm{d} v \mathrm{~d} u \\
& =\int_{W_{n} \oplus \breve{W}_{n}} \operatorname{cov}_{Y}(x) \int_{W_{n} \cap\left(W_{n}-x\right)} G\left(W_{n}, y\right) G\left(W_{n}, x+y\right) \mathrm{d} y \mathrm{~d} x \\
& =\int_{\mathbb{R}^{d}} \operatorname{cov}_{Y}(x) R_{W_{n}}(x) \mathrm{d} x,
\end{aligned}
$$

where the last equality follows from the fact that $W_{n} \cap\left(W_{n}-x\right)=\varnothing$ for any $x \notin W_{n} \oplus \check{W}_{n}$.

To determine the asymptotic behavior of the estimation variance $\operatorname{var}\left(\hat{\mu}_{n}\right)$, we need some further conditions on the weighting function $G: \mathcal{B}_{0}\left(\mathbb{R}^{d}\right) \times \mathbb{R}^{d} \rightarrow[0, \infty)$. Besides (2.8), we additionally assume that there exist constants $c_{1}, c_{2}<\infty$ such that

$$
\begin{aligned}
& \sup _{y \in W_{n}} G\left(W_{n}, y\right) \leq \frac{c_{1}}{\left|W_{n}\right|} \quad \text { for any } n \geq 1, \\
& \lim _{n \rightarrow \infty}\left|W_{n}\right| R_{W_{n}}(x)=c_{2} \quad \text { for any } x \in \mathbb{R}^{d} \text {. }
\end{aligned}
$$

Notice that (2.8) and (2.9) hold, for example, if $G\left(W_{n}, x\right)=\mathbf{1}\left(x \in W_{n} \ominus \check{K}\right) /\left|W_{n} \ominus \check{K}\right|$ for any $n \geq 1$ and $x \in \mathbb{R}^{d}$, where $\mathbf{1}(B)$ denotes the indicator function of event $B$. In this case, from (2.7) we have $c_{1}=2$ and $c_{2}=1$. Furthermore, we assume that the covariance $\operatorname{cov}_{Y}(x)$ of the stationary random field $Y$ is integrable, i.e. that

$$
\int_{\mathbb{R}^{d}}\left|\operatorname{cov}_{Y}(x)\right| \mathrm{d} x<\infty .
$$

Lemma 2.3. Let the conditions (2.6), (2.8), (2.9), and (2.10) be fulfilled. Then

$$
\lim _{n \rightarrow \infty}\left|W_{n}\right| \operatorname{var}\left(\hat{\mu}_{n}\right)=c_{2} \int_{\mathbb{R}^{d}} \operatorname{cov}_{Y}(x) \mathrm{d} x .
$$

Proof. Conditions (2.8) and (2.9) immediately imply that $\left|W_{n}\right| R_{W_{n}}(x) \leq c_{1}$ holds for any $x \in \mathbb{R}^{d}$ and $n \geq 1$. Thus, using Lemma 2.2 and condition (2.10), the assertion follows from the Lebesgue dominated convergence theorem.

Since $\lim _{n \rightarrow \infty}\left|W_{n}\right|=\infty$, Lemma 2.3 implies, in particular, that $\lim _{n \rightarrow \infty} \operatorname{var}\left(\hat{\mu}_{n}\right)=0$, i.e. that the unbiased estimator $\hat{\mu}_{n}$ is also mean-square consistent for $\mu$. 


\section{Examples}

In this section, we briefly discuss some examples of stationary random fields that belong to the general class of random fields $Y=\left\{Y(x), x \in \mathbb{R}^{d}\right\}$ introduced in (2.5). They can be used to construct unbiased and mean-square consistent estimators for various morphological characteristics of stationary RACS. In the sequel, we assume that $\left\{W_{n}\right\}$ is an arbitrary sequence of bounded Borel sets that satisfies (2.6), with $\left|W_{n}\right|>0$ for any $n \geq 1$.

\subsection{Volume fraction}

Let $\Xi$ be a stationary RACS in $\mathbb{R}^{d}$ with volume fraction $p=\mathrm{P}(o \in \Xi)$, and let $Z_{d}=$ $\left\{Z_{d}(x), x \in \mathbb{R}^{d}\right\}$ be the random field given by $Z_{d}(x)=\mathbf{1}(x \in \Xi)$. Then

$$
\hat{p}_{n}=\frac{1}{\left|W_{n}\right|} \int_{W_{n}} Z_{d}(x) \mathrm{d} x
$$

is an unbiased estimator for $p$. Since $\mathbf{1}(x \in \Xi)=\mathbf{1}((\Xi-x) \cap\{o\} \neq \varnothing)$ for any $x \in \mathbb{R}^{d}$, it is easy to see that $Z_{d}$ is of the form considered in (2.5) with $K=\{o\}$ and the (bounded) valuation $f: \mathcal{R} \rightarrow \mathbb{R}$ given by $f\left(K^{\prime}\right)=\mathbf{1}\left(K^{\prime} \neq \varnothing\right)$. Clearly, the random field $Z_{d}$ is of second order. If $\Xi=\bigcup_{i=1}^{\infty}\left(M_{i}+X_{i}\right)$ is a Boolean model with $\mathrm{E}\left(\left|M_{0}\right|^{2}\right)<\infty$, then it is well known that the covariance $\operatorname{cov}_{Z_{d}}(x)$ of $Z_{d}$ is integrable; see, e.g. the remarks following Corollary 4.2 of [1]. According to Lemma 2.3, the unbiased estimator $\hat{p}_{n}$ is mean-square consistent for $p$.

\subsection{Specific intrinsic volumes}

Let $\Xi$ be a stationary RACS such that $\Xi \in \delta$ holds with probability 1 . Then, for each $i=0, \ldots, d$, the intrinsic volume $V_{i}(\Xi \cap K)$ of $\Xi \cap K$ is well defined for any convex body $K \in \mathcal{K}$, where, for instance, $V_{d}(\Xi \cap K)=|\Xi \cap K|$ is the usual volume, $V_{0}(\Xi \cap K)$ is the Euler number of the set $\Xi \cap K$, which is defined by the inclusion-exclusion formula (2.4), and $V_{0}(M)=\mathbf{1}(M \neq \varnothing), M \in \mathcal{K} ;$ see also [12].

Assume that $\mathrm{E}\left(2^{\tilde{N}\left(\Xi \cap[0,1]^{d}\right)}\right)<\infty$, where $\tilde{N}(B)$ denotes the minimal number of convex components of the polyconvex set $B \in \mathcal{R}$. Then, for any sequence $\left\{K_{n}\right\}$ of convex bodies $K_{n}=n K_{0}$, with $K_{0} \in \mathcal{K}$ such that $\left|K_{0}\right|>0$ and $o \in \operatorname{int}\left(K_{0}\right)$, the limits

$$
\bar{V}_{i}(\Xi)=\lim _{n \rightarrow \infty} \frac{\mathrm{E}\left(V_{i}\left(\Xi \cap K_{n}\right)\right)}{\left|K_{n}\right|}, \quad i=0, \ldots, d,
$$

exist and are called the specific intrinsic volumes of $\Xi$; see, e.g. [13, Section 5.1, pp. 185-187]. Estimators of several types for some specific intrinsic volumes have been considered in the literature. Two indirect estimation methods were proposed in [11] and [14], respectively. These have the advantage that joint estimators can be constructed for the vector $\left(\bar{V}_{0}(\Xi), \ldots, \bar{V}_{d}(\Xi)\right)$ of all $d+1$ specific intrinsic volumes.

The construction principle considered in [11] (see Section 2.3 thereof) is based on Steiner's formula and makes use of the index of polyconvex sets. The random field used therein is defined as follows. For $i=0, \ldots, d-1$, let $r_{i}>0$ be any positive number and let the random field $Z_{i}=\left\{Z_{i}(x), x \in \mathbb{R}^{d}\right\}$ be given by

$$
Z_{i}(x)=\sum_{q \in \partial\left((\Xi-x) \cap B_{r_{i}}(o)\right), q \neq 0} J\left((\Xi-x) \cap B_{r_{i}}(o), q, o\right),
$$

where the functional

$$
J(K, q, x)=\mathbf{1}(q \in K)\left(1-\lim _{\delta \rightarrow+0} \lim _{\varepsilon \rightarrow+0} V_{0}\left(K \cap B_{|x-q|-\varepsilon}(x) \cap B_{\delta}(q)\right)\right)
$$


is called the index of $K \in \mathcal{R}$ at $x \in \mathbb{R}^{d}$. It is not difficult to see that $Z_{i}$ is of the form considered in (2.5) with $K=B_{r_{i}}(o)$ and the valuation $f: \mathcal{R} \rightarrow \mathbb{R}$ given by

$$
f\left(K^{\prime}\right)=\sum_{q \in \partial K^{\prime}, q \neq 0} J\left(K^{\prime}, q, o\right) .
$$

Here, the functional $f$ is bounded on $\mathcal{K}$, with $f\left(K^{\prime}\right)=\mathbf{1}\left(o \notin K^{\prime}, K^{\prime} \neq \varnothing\right)$ for any $K^{\prime} \in \mathcal{K}$. If the covariance $\operatorname{cov}_{Z_{i}}(x)$ of $Z_{i}$ is integrable for any $i=0, \ldots, d$, it can be concluded from Lemma 2.3 that

$$
\hat{\mu}_{n, i}=\left|W_{n} \ominus B_{r_{i}}(o)\right|^{-1} \int_{W_{n}} Z_{i}(x) \mathbf{1}\left(x \in W_{n} \ominus B_{r_{i}}(o)\right) \mathrm{d} x
$$

is an unbiased estimator for $\mu_{i}=\mathrm{E}\left(Z_{i}(o)\right)$ that is mean-square consistent provided that $\left\{W_{n}\right\}$ satisfies (2.6). Then, assuming that $r_{i} \neq r_{i^{\prime}}$ for any $i \neq i^{\prime}$, the random vector $\hat{v}_{n}=$ $A_{r_{0}, \ldots, r_{d-1}}^{-1}\left(\hat{\mu}_{n, 0}, \ldots, \hat{\mu}_{n, d-1}, \hat{p}_{n}\right)^{\top}$, where $\hat{p}_{n}$ is the empirical volume fraction introduced in (3.1) and $A_{r_{0}, \ldots, r_{d-1}}$ is a regular matrix of Vandermond type (see [11]), provides an unbiased, mean-square consistent estimator for $\left(\bar{V}_{0}(\Xi), \ldots, \bar{V}_{d}(\Xi)\right)$.

The estimator proposed in [14] employs the principal kinematic formula. Here, the construction principle is as follows. For $i=0, \ldots, d$, introduce the random fields $\tilde{Z}_{i}=\left\{\tilde{Z}_{i}(x)\right.$, $\left.x \in \mathbb{R}^{d}\right\}$ with $\tilde{Z}_{i}(x)=V_{0}\left((\Xi-x) \cap B_{r_{i}}(o)\right)$. Each $\tilde{Z}_{i}$ is of the form (2.5) with $f\left(K^{\prime}\right)=V_{0}\left(K^{\prime}\right)$, $K^{\prime} \in \mathcal{R}$, and $K=B_{r_{i}}(0)$, where $f$ is bounded on $\mathcal{K}$ with $f\left(K^{\prime}\right)=\mathbf{1}\left(K^{\prime} \neq \varnothing\right)$ for all $K^{\prime} \in \mathcal{K}$. For any $d+1$ pairwise-different positive radii $r_{0}, \ldots, r_{d}$, define

$$
\tilde{\mu}_{n, i}=\int_{W_{n} \ominus B_{r_{i}}(o)} \frac{\tilde{Z}_{i}(x)}{\left|W_{n} \ominus B_{r_{i}}(o)\right|} \mathrm{d} x
$$

and

$$
\tilde{A}_{r_{0}, \ldots, r_{d}}=\left(\begin{array}{cccccc}
r_{0}^{d} \kappa_{d} & r_{0}^{d-1} \kappa_{d-1} & \cdots & r_{0}^{2} \kappa_{2} & r_{0} \kappa_{1} & 1 \\
r_{1}^{d} \kappa_{d} & r_{1}^{d-1} \kappa_{d-1} & \cdots & r_{1}^{2} \kappa_{2} & r_{1} \kappa_{1} & 1 \\
\vdots & \vdots & \ddots & \vdots & \vdots & \vdots \\
r_{d}^{d} \kappa_{d} & r_{d}^{d-1} \kappa_{d-1} & \cdots & r_{d}^{2} \kappa_{2} & r_{d} \kappa_{1} & 1
\end{array}\right),
$$

where $\kappa_{i}$ denotes the volume of the unit ball in $\mathbb{R}^{i}, i=1, \ldots, d$. Then the random vector $\tilde{v}_{n}=\tilde{A}_{r_{0}, \ldots, r_{d}}^{-1}\left(\tilde{\mu}_{n, 0}, \ldots, \tilde{\mu}_{n, d}\right)^{\top}$ is an unbiased, mean-square consistent estimator for $\left(\bar{V}_{0}(\Xi), \ldots, \bar{V}_{d}(\Xi)\right)$.

\section{Asymptotic normality for functionals of Boolean models}

Let $\Xi=\bigcup_{i=1}^{\infty}\left(M_{i}+X_{i}\right)$ be a Boolean model with compact and convex typical grain $M_{0} \in \mathcal{K}^{\prime}=\mathcal{K} \backslash\{\varnothing\}$. The aim of this section is to prove asymptotic normality with respect to $\hat{\mu}_{n}=\int_{W_{n}} Y(x) G\left(W_{n}, x\right) \mathrm{d} x$, which is an estimator for the mean value, $\mu$, of the random field $Y$ introduced in (2.5). We thus assume that the conditions (2.6), (2.8), (2.9), and (2.10) are fulfilled. More precisely, by replacing (2.10) by a moment condition on $M_{0}$, we show that

$$
\sqrt{\left|W_{n}\right|}\left(\hat{\mu}_{n}-\mu\right) \stackrel{\mathrm{D}}{\rightarrow} \mathcal{N}\left(0, \sigma^{2}\right), \quad n \rightarrow \infty
$$

where ' $\stackrel{\mathrm{D}}{\rightarrow}$ ' denotes convergence in distribution and $\mathcal{N}\left(0, \sigma^{2}\right)$ is a Gaussian random variable with mean 0 and variance $\sigma^{2}=c_{2} \int_{\mathbb{R}^{d}} \operatorname{cov}_{Y}(x) \mathrm{d} x$. 
We concentrate on the case of the Boolean model for two reasons. First, the integrability of

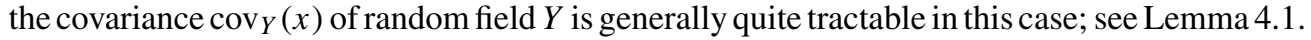
Second, we can make use of a central limit theorem for $m$-dependent random fields, from [4], without imposing further conditions. A corresponding central limit theorem for more general germ-grain models is considered in Section 5.

\subsection{Integrability of the covariance}

The following lemma yields a simple condition sufficient for absolute integrability of the covariance $\operatorname{cov}_{Y}(x), x \in \mathbb{R}^{d}$.

Lemma 4.1. Assume that $\mathrm{E}\left(\left|M_{0} \oplus \check{K}\right|^{2}\right)<\infty$. Then (2.10) holds.

Proof. For better readability, we use the representation of $\Xi$ as the set-theoretic union of the generating Poisson particle process $\tilde{M}=\left\{\tilde{M}_{i}\right\}$ in $\mathcal{K}^{\prime}$ with $\tilde{M}_{i}=M_{i}+X_{i}$, and let $\Lambda$ denote the intensity measure of $\tilde{M}$. By Campbell's theorem for independently marked point processes on $\mathbb{R}^{d}$ (see, e.g. Section 3 of [13, pp. 66, 93]), the following representation for $\Lambda$ holds for any set $B \subseteq \mathcal{K}$ with $B \in \sigma_{\mathcal{F}}$, where $\mathbb{Q}$ denotes the distribution of the typical grain $M_{0}$ :

$$
\Lambda(B)=\lambda \int_{\mathcal{K}} \int_{\mathbb{R}^{d}} \mathbf{1}\left(\left(M_{0}+y\right) \in B\right) \mathrm{d} y \mathrm{~d} \mathbb{Q}\left(M_{0}\right) .
$$

Now, let $\mathcal{K}_{x}^{*}=\mathcal{K}_{K} \cap \mathcal{K}_{K+x}$, with $\mathcal{K}_{K}=\left\{K^{\prime} \in \mathcal{K}: K^{\prime} \cap K \neq \varnothing\right\}$ for any set $K \in \mathcal{K}^{\prime}$, and let $B \triangle B^{\prime}=\left(B \cup B^{\prime}\right) \backslash\left(B \cap B^{\prime}\right)$ be the symmetric difference between any two sets $B$ and $B^{\prime}$. Considering the event $A=\left\{\tilde{M}\left(\mathcal{K}_{x}^{*}\right)>0\right\}$ and its complement $A^{\text {c }}$, where $\tilde{M}(B)$ is the number of particles of $\tilde{M}$ in a set $B \subseteq \mathcal{K}$, we can write

$$
\operatorname{cov}_{Y}(x)=\mathrm{E}(Y(o)(Y(x)-\mu))=\mathrm{E}(Y(o) \mathbf{1}(A)(Y(x)-\mu))+\mathrm{E}\left(Y(o) \mathbf{1}\left(A^{\mathrm{c}}\right)(Y(x)-\mu)\right) .
$$

Using arguments similar to those in the proof of Lemma 2.1, upper bounds for the absolute values of the summands in the above decomposition of $\operatorname{cov}_{Y}(x)$ can be deduced in the following way. We have

$$
\begin{aligned}
|\mathrm{E}(Y(o) \mathbf{1}(A)(Y(x)-\mu))| \leq & c^{2}(K) \mathrm{E}\left(2^{\tilde{M}\left(\mathcal{K}_{K}\right)+\tilde{M}\left(\mathcal{K}_{K+x}\right)} \mathbf{1}(A)\right)+c(K)|\mu| \mathrm{E}\left(2^{\tilde{M}\left(\mathcal{K}_{K}\right)} \mathbf{1}(A)\right) \\
= & c^{2}(K) \mathrm{E}\left(2^{\tilde{M}\left(\mathcal{K}_{K} \triangle \mathcal{K}_{K+x}\right)}\right) \mathrm{E}\left(2^{2 \tilde{M}\left(\mathcal{K}_{x}^{*}\right)} \mathbf{1}(A)\right) \\
& +c(K)|\mu| \mathrm{E}\left(2^{\tilde{M}\left(\mathcal{K}_{K} \backslash \mathcal{K}_{x}^{*}\right)}\right) \mathrm{E}\left(2^{\tilde{M}\left(\mathcal{K}_{x}^{*}\right)} \mathbf{1}(A)\right) \\
\leq & 2 c^{2}(K) \mathrm{E}\left(2^{\tilde{M}\left(\mathcal{K}_{K}\right)}\right)^{2} \mathrm{E}\left(4^{\tilde{M}\left(\mathcal{K}_{x}^{*}\right)} \mathbf{1}(A)\right),
\end{aligned}
$$

since the random variables $2^{\tilde{M}\left(\mathcal{K}_{K} \Delta \mathcal{K}_{K+x}\right)}$ and $2^{\tilde{M}\left(\mathcal{K}_{K} \backslash \mathcal{K}_{x}^{*}\right)}$ are independent of $4^{\tilde{M}\left(\mathcal{K}_{x}^{*}\right)} \mathbf{1}(A)$, where we have employed Lemma 2.1 and the stationarity of $\tilde{M}$. Now consider the second summand of the representation of $\operatorname{cov}(x)$, and define $Y_{B}(x)=f\left(\bigcup_{\left\{i: \tilde{M}_{i} \in B\right\}}\left(\tilde{M}_{i}-x\right) \cap K\right)$ for any $B \subseteq \mathcal{K}$. From the properties of the valuation $f$, it follows that $Y(o) \mathbf{1}\left(A^{\mathrm{c}}\right)=$

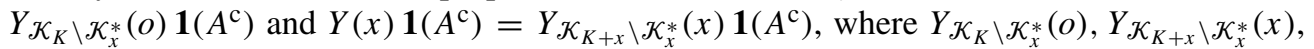
and $\mathbf{1}\left(A^{\mathrm{c}}\right)$ are mutually independent. Hence, we have

$$
\begin{aligned}
& \left|\mathrm{E}\left(Y(o) \mathbf{1}\left(A^{\mathrm{c}}\right)(Y(x)-\mu)\right)\right|=\left|\mathrm{E}\left(Y_{\mathcal{K}_{K} \backslash \mathcal{K}_{x}^{*}}(o) \mathbf{1}\left(A^{\mathrm{c}}\right)\left(Y_{\mathcal{K}_{K+x} \backslash \mathcal{K}_{x}^{*}}(x)-\mu\right)\right)\right| \\
& =\left|\mathrm{E}\left(Y(o) \mathbf{1}\left(A^{\mathrm{c}}\right)\right)\right|\left|\mathrm{E}\left(Y_{\mathcal{K}_{K+x} \backslash \mathcal{K}_{x}^{*}}(x)-\mu\right)\right| \\
& =\left|\mathrm{E}\left(Y(o) \mathbf{1}\left(A^{\mathrm{c}}\right)\right)\right|\left|\mathrm{E}\left(\left(Y_{\mathcal{K}_{K+x} \backslash \mathcal{K}_{x}^{*}}(x)-Y(x)\right) \mathbf{1}(A)\right)\right| \\
& \leq c(K) \mathrm{E}(|Y(o)|) \mathrm{E}\left(2^{\tilde{M}\left(\mathcal{K}_{K}\right)}\right)\left[\mathrm{E}(\mathbf{1}(A))+\mathrm{E}\left(2^{\tilde{M}\left(\mathcal{K}_{x}^{*}\right)} \mathbf{1}(A)\right)\right],
\end{aligned}
$$


where the inequality follows as before. Notice that $\mathrm{E}\left(s^{\tilde{M}\left(\mathcal{K}_{K}\right)}\right)=\mathrm{e}^{(s-1) \Lambda\left(\mathcal{K}_{K}\right)}<\infty$ for any $s \in \mathbb{R}$, since $\Lambda\left(\mathcal{K}_{K}\right)=\lambda \mathrm{E}\left(\left|M_{0} \oplus \check{K}\right|\right)<\infty$ by condition (2.2) and (4.2). Thus, it suffices to show that $\mathrm{E}\left(4^{\tilde{M}\left(\mathcal{K}_{x}^{*}\right)} \mathbf{1}(A)\right)$ is integrable with respect to $x \in \mathbb{R}^{d}$. Observe that, since $\tilde{M}$ is Poisson,

$\mathrm{E}\left(4^{\tilde{M}\left(\mathcal{K}_{x}^{*}\right)} \mathbf{1}(A)\right)=\mathrm{E}\left(4^{\tilde{M}\left(\mathcal{K}_{x}^{*}\right)}\right)-\mathrm{E}\left(\mathbf{1}\left(A^{\mathrm{c}}\right)\right)=\mathrm{e}^{3 \Lambda\left(\mathcal{K}_{x}^{*}\right)}\left(1-\mathrm{e}^{-4 \Lambda\left(\mathcal{K}_{x}^{*}\right)}\right) \leq 4 \mathrm{e}^{3 \lambda \mathrm{E}\left(\left|M_{0} \oplus \check{K}\right|\right)} \Lambda\left(\mathcal{K}_{x}^{*}\right)$,

where we have used the estimate $1-\mathrm{e}^{-s} \leq s$ for any $s \geq 0$ to obtain the latter inequality. By virtue of Campbell's formula and Fubini's formula, we can finally conclude that

$$
\begin{aligned}
\int_{\mathbb{R}^{d}} \Lambda\left(\mathcal{K}_{x}^{*}\right) \mathrm{d} x & =\int_{\mathbb{R}^{d}} \mathrm{E}\left(\sum_{i=1}^{\infty} \mathbf{1}\left(\left(M_{i}+X_{i}\right) \cap K \neq \varnothing,\left(M_{i}+X_{i}\right) \cap(K+x) \neq \varnothing\right)\right) \mathrm{d} x \\
& =\int_{\mathbb{R}^{d}} \lambda \mathrm{E}\left(\int_{\mathbb{R}^{d}} \mathbf{1}\left(\left(M_{0}+y\right) \cap K \neq \varnothing,\left(M_{0}+y\right) \cap(K+x) \neq \varnothing\right) \mathrm{d} y\right) \mathrm{d} x \\
& =\lambda \mathrm{E}\left(\int_{\mathbb{R}^{d}} \int_{\mathbb{R}^{d}} \mathbf{1}\left(y \in\left(\check{M}_{0} \oplus K\right)\right) \mathbf{1}\left((y-x) \in\left(\check{M}_{0} \oplus K\right)\right) \mathrm{d} y \mathrm{~d} x\right) \\
& =\lambda \mathrm{E}\left(\left|\check{M}_{0} \oplus K\right|^{2}\right)=\lambda \mathrm{E}\left(\left|M_{0} \oplus \check{K}\right|^{2}\right)<\infty .
\end{aligned}
$$

Note that the proof of Lemma 4.1 provides an integrable upper bound $h(x) \equiv h\left(x, M_{0}\right)$ for $\left|\operatorname{cov}_{Y}(x)\right|$ that depends on the distribution of the typical grain $M_{0}$. This dependence is monotone with respect to set inclusion. That is, if $M_{0}^{(1)} \subseteq M_{0}^{(2)}$ then with probability 1 $h\left(x, M_{0}^{(1)}\right) \leq h\left(x, M_{0}^{(2)}\right)$ for any $x \in \mathbb{R}^{d}$.

\subsection{Truncated germ-grain models}

Let the conditions (2.6), (2.8), and (2.9) be fulfilled and assume that $\mathrm{E}\left(\left|M_{0} \oplus \check{K}\right|^{2}\right)<\infty$. To prove the central limit theorem (4.1), we approximate the random field $Y$ corresponding to $\Xi$ by random fields $Y_{n}$ that are induced by germ-grain models $\Xi_{n}$ with truncated grains. These are chosen in the following way. For any $n \geq 1$, let $A_{n}=\left[-a_{n}, a_{n}\right]^{d}$ for some $a_{n}>0$ such that $\lim _{n \rightarrow \infty} a_{n}=\infty$. Introduce the auxiliary germ-grain model $\Xi_{n}$ defined by

$$
\Xi_{n}=\bigcup_{i=1}^{\infty}\left(M_{n, i}+X_{i}\right),
$$

where $M_{n, i}=M_{i} \cap A_{n} \in \mathcal{K}$ for any $i, n \in \mathbb{N}$. Accordingly, define the random field $Y_{n}=\left\{Y_{n}(x), x \in \mathbb{R}^{d}\right\}$ by $Y_{n}(x)=f\left(\left(\Xi_{n}-x\right) \cap K\right)$, and let $\mu_{n}=\mathrm{E}\left(Y_{n}(o)\right)$ and $\hat{\mu}_{n}^{\prime}=$ $\int_{W_{n}} Y_{n}(x) G\left(W_{n}, x\right) \mathrm{d} x$.

Lemma 4.2. The random fields $Y$ and $Y_{n}$ are of second order. Moreover, $Y_{n}(x)$ converges in mean square to $Y(x)$ as $n \rightarrow \infty$, i.e. $\lim _{n \rightarrow \infty} \mathrm{E}\left(\left|Y(x)-Y_{n}(x)\right|^{2}\right)=0$ for all $x \in \mathbb{R}^{d}$.

Proof. Due to stationarity, we can assume that $x=o$. Since $g_{N\left(\Xi_{n} \cap K\right)}(4) \leq g_{N(\Xi \cap K)}(4)<$ $\infty$, the random fields $Y$ and $Y_{n}$ are both of second order, by Lemma 2.1. To prove the second assertion, we let

$$
N_{\mid A_{n}}(\Xi \cap K)=\operatorname{card}\left\{i:\left(M_{i}+X_{i}\right) \cap K \neq \varnothing, K \nsubseteq\left(A_{n}+X_{i}\right)\right\} .
$$

Then, using arguments similar to those in the proof of Lemma 2.1, we have

$$
\mathrm{E}\left(\left|Y(o)-Y_{n}(o)\right|^{2}\right) \leq 4 c^{2}(K) \mathrm{E}\left(2^{2 N(\Xi \cap K)} \mathbf{1}\left(N_{\mid A_{n}}(\Xi \cap K)>0\right)\right) .
$$


Since $\mathrm{E}\left(2^{2 N(\Xi \cap K)}\right)=\mathrm{e}^{3 \lambda \mathrm{E}\left(\left|M_{0} \oplus \check{K}\right|\right)}<\infty$, it now suffices to show that

$$
\lim _{n \rightarrow \infty} \mathrm{P}\left(N_{\mid A_{n}}(\Xi \cap K)>0\right)=0 .
$$

Campbell's formula and Fubini's theorem yield

$$
\begin{aligned}
\mathrm{P}\left(N_{\mid A_{n}}(\Xi \cap K)>0\right) & \leq \mathrm{E}\left(N_{\mid A_{n}}(\Xi \cap K)\right) \\
& =\lambda \mathrm{E}\left(\int_{\mathbb{R}^{d}} \mathbf{1}\left(\left(M_{0}+y\right) \cap K \neq \varnothing, K \not\left(A_{n}+y\right)\right) \mathrm{d} y\right) \\
& \leq \lambda \mathrm{E} \int_{\mathbb{R}^{d}} \mathbf{1}\left(y \in\left(M_{0} \oplus \check{K}\right)\right) \mathbf{1}\left(y \notin\left(A_{n} \ominus \check{K}\right)\right) \mathrm{d} y \\
& \leq \lambda \mathrm{E} \int_{\mathbb{R}^{d}} \mathbf{1}\left(y \in\left(M_{0} \oplus \check{K}\right)\right) \mathbf{1}\left(|y|>a_{n}-D(K)\right) \mathrm{d} y
\end{aligned}
$$

for any $n$ large enough that $a_{n}-D(K)>0$. By the dominated convergence theorem, the final expression on the right-hand side converges to 0 as $n \rightarrow \infty$, since $\lim _{n \rightarrow \infty} a_{n}=\infty$ and $\mathrm{E}\left(\left|M_{0} \oplus \check{K}\right|\right)<\infty$.

Next, we show that the asymptotic variance of the estimator $\hat{\mu}_{n}^{\prime}=\int_{W_{n}} Y_{n}(x) G\left(W_{n}, x\right) \mathrm{d} x$ of $\mu_{n}$ is equal to the asymptotic variance of the estimator $\hat{\mu}_{n}=\int_{W_{n}} Y(x) G\left(W_{n}, x\right) \mathrm{d} x$ of the mean of $Y$.

Lemma 4.3. The covariance $\operatorname{cov}_{Y_{n}}(x)$ of the stationary random field $Y_{n}$ is integrable and

$$
\lim _{n \rightarrow \infty}\left|W_{n}\right| \operatorname{var}\left(\hat{\mu}_{n}^{\prime}\right)=c_{2} \int_{\mathbb{R}^{d}} \operatorname{cov}_{Y}(x) \mathrm{d} x,
$$

where the constant $c_{2}>0$ is as defined in (2.9).

Proof. The integrability of $\operatorname{cov}_{Y_{n}}(x)$ immediately follows from Lemma 4.1. By Lemma 2.2, we have $\left|W_{n}\right| \operatorname{var}\left(\hat{\mu}_{n}^{\prime}\right)=\int_{\mathbb{R}^{d}} \operatorname{cov}_{Y_{n}}(x)\left|W_{n}\right| R_{W_{n}}(x) \mathrm{d} x$, where $\lim _{n \rightarrow \infty}\left|W_{n}\right| R_{W_{n}}(x)=c_{2}$ by (2.9). As mentioned above, there exists an integrable function $h: \mathbb{R}^{d} \times \mathcal{K} \rightarrow \mathbb{R}_{+}$such that $\left|\operatorname{cov}_{Y_{n}}(x)\right| \leq h\left(x, M_{0} \cap A_{n}\right) \leq h\left(x, M_{0}\right)$ for any $x \in \mathbb{R}^{d}$ and $n \in \mathbb{N}$. Together with (2.9), this implies that $\left|\operatorname{cov}_{Y_{n}}(x)\right|\left|W_{n}\right| R_{W_{n}}(x) \leq c_{1} h\left(x, M_{0}\right)$ for all $x \in \mathbb{R}^{d}$. Furthermore, we have $\lim _{n \rightarrow \infty} \operatorname{cov}_{Y_{n}}(x)=\operatorname{cov}_{Y}(x)$, since

$$
\begin{aligned}
\left|\operatorname{cov}_{Y_{n}}(x)-\operatorname{cov}_{Y}(x)\right| & \leq\left|\mathrm{E}\left(Y_{n}(o) Y_{n}(x)\right)-\mathrm{E}(Y(o) Y(x))\right|+\left|\mu_{n}^{2}-\mu^{2}\right| \\
& =\left|\mathrm{E}\left(\left(Y_{n}(o)-Y(o)\right) Y_{n}(x)\right)+\mathrm{E}\left(\left(Y_{n}(x)-Y(x)\right) Y(o)\right)\right|+\left|\mu_{n}^{2}-\mu^{2}\right| \\
& \leq \mathrm{E}\left(\left(Y_{n}(o)-Y(o)\right)^{2}\right)^{1 / 2}\left[\mathrm{E}\left(Y_{n}^{2}(o)\right)^{1 / 2}+\mathrm{E}\left(Y^{2}(o)\right)^{1 / 2}\right]+\left|\mu_{n}^{2}-\mu^{2}\right|,
\end{aligned}
$$

with $\lim _{n \rightarrow \infty} \mathrm{E}\left(\left(Y_{n}(o)-Y(o)\right)^{2}\right)=0$ and $\lim _{n \rightarrow \infty}\left|\mu_{n}^{2}-\mu^{2}\right|=0$ by Lemma 4.2. Furthermore, $\mathrm{E}\left(Y_{n}^{2}(o)\right)$ and $\mathrm{E}\left(Y^{2}(o)\right)$ are uniformly bounded in $n$. Consequently, the limit in (4.3) follows from the dominated convergence theorem.

Let $\|z\|=\max \left\{\left|z_{i}\right|, i=1, \ldots, d\right\}$ for any $z=\left(z_{1}, \ldots, z_{d}\right) \in \mathbb{Z}^{d}$, and let $m>0$ be an arbitrary integer. A family of random variables $\left\{Z_{z}, z \in \mathbb{Z}^{d}\right\}$ is called an $m$-dependent random field if $\left(Z_{z}\right)_{z \in U}$ and $\left(Z_{z}\right)_{z \in U^{\prime}}$ are independent random vectors for any finite sets $U, U^{\prime} \subset \mathbb{Z}^{d}$ with $\inf \left\{\left\|z-z^{\prime}\right\|, z \in U, z^{\prime} \in U^{\prime}\right\}>m$. Any stationary random field indexed over $\mathbb{Z}^{d}$ that satisfies an appropriate $\beta$-mixing condition is $m$-dependent; see, e.g. Section 1.3.1 of [2, p. 17].

The following central limit theorem for $\hat{\mu}_{n}^{\prime}$ is closely related to the Lindeberg-type central limit theorem for $m$-dependent random fields presented in Theorem 2 of [4]. 
Lemma 4.4. Let $\left\{m_{n}, n \geq 1\right\}$ be an arbitrary sequence of positive integers such that $m_{n} \rightarrow \infty$, and let $\left\{U_{n}, n \geq 1\right\}$ be a sequence of finite subsets of $\mathbb{Z}^{d}$ with $\lim _{n \rightarrow \infty} \operatorname{card}\left(U_{n}\right)=\infty$. For each $n \geq 1$, let $\left\{Z_{n, z}, z \in \mathbb{Z}^{d}\right\}$ be an $m_{n}$-dependent random field with $\mathrm{E}\left(Z_{n, z}\right)=0$ for any $z \in U_{n}$ and $\mathrm{E}\left(S_{n}^{*}\right)^{2}=\sigma_{n}^{2} \rightarrow \sigma^{2}$ as $n \rightarrow \infty$, where $S_{n}^{*}=\sum_{z \in U_{n}} Z_{n, z}$. If there exists a constant $c>0$ such that

$$
\sum_{z \in U_{n}} \mathrm{E}\left(Z_{n, z}^{2}\right) \leq c
$$

for any $n \geq 1$, and if

$$
\lim _{n \rightarrow \infty} m_{n}^{2 d} \sum_{z \in U_{n}} \mathrm{E}\left(Z_{n, z}^{2} \mathbf{1}\left(\left|Z_{n z}\right| \geq \varepsilon m_{n}^{-2 d}\right)\right)=0
$$

for any $\varepsilon>0$, then $S_{n}^{*} \stackrel{\mathrm{D}}{\rightarrow} \mathcal{N}\left(0, \sigma^{2}\right)$ as $n \rightarrow \infty$.

The proof of Lemma 4.4 for $\sigma_{n}^{2}=1$ can be found in, e.g. Section 3 of [4] and extended easily to the case in which $\lim _{n \rightarrow \infty} \sigma_{n}^{2}=\sigma^{2}<\infty$.

Lemma 4.5. If the truncation sequence $\left\{a_{n}\right\}$ satisfies

$$
\lim _{n \rightarrow \infty} a_{n}=\infty \text { and } \lim _{n \rightarrow \infty} \frac{a_{n}^{4 d(1+\delta) / \delta}}{\left|W_{n}\right|}=0
$$

for some $\delta>0$, then the random variables $S_{n}^{\prime}=\sqrt{\left|W_{n}\right|}\left(\hat{\mu}_{n}^{\prime}-\mu_{n}\right)$ are asymptotically normally distributed, i.e.

$$
S_{n}^{\prime} \stackrel{\mathrm{D}}{\rightarrow} \mathcal{N}\left(0, \sigma^{2}\right) \text { as } n \rightarrow \infty,
$$

where $\sigma^{2}=c_{2} \int_{\mathbb{R}^{d}} \operatorname{cov}_{Y}(x) \mathrm{d} x$ with $c_{2}$ as defined in (2.9).

Proof. Let $[z, z+e)=\left[z_{1}, z_{1}+1\right) \times \cdots \times\left[z_{d}, z_{d}+1\right)$ for $z \in \mathbb{Z}^{d}$, and consider the sets $U_{n}=\left\{z \in \mathbb{Z}^{d}:[z, z+e) \subseteq W_{n}\right\}$ and $W_{n}^{-}=\bigcup_{z \in U_{n}}[z, z+e)$. Furthermore, decompose $S_{n}^{\prime}$ into $S_{n}^{\prime}=S_{n}^{*}+\tilde{S}_{n}$, with

$$
\begin{aligned}
& S_{n}^{*}=\sqrt{\left|W_{n}\right|} \int_{W_{n}^{-}}\left(Y_{n}(x)-\mu_{n}\right) G\left(W_{n}, x\right) \mathrm{d} x, \\
& \tilde{S}_{n}=\sqrt{\left|W_{n}\right|} \int_{W_{n} \backslash W_{n}^{-}}\left(Y_{n}(x)-\mu_{n}\right) G\left(W_{n}, x\right) \mathrm{d} x .
\end{aligned}
$$

Condition (2.9) and the integrability of $\operatorname{cov}_{Y_{n}}(x)$ imply that

$$
\begin{aligned}
\lim _{n \rightarrow \infty} \mathrm{E}\left(\tilde{S}_{n}^{2}\right) \\
=\lim _{n \rightarrow \infty} \int_{W_{n} \backslash W_{n}^{-}} \int_{W_{n} \backslash W_{n}^{-}}\left|W_{n}\right| \mathrm{E}\left(\left(Y_{n}(x)-\mu_{n}\right)\left(Y_{n}(y)-\mu_{n}\right)\right) G\left(W_{n}, x\right) G\left(W_{n}, y\right) \mathrm{d} x \mathrm{~d} y \\
=\lim _{n \rightarrow \infty} \int_{\mathbb{R}^{d}} \operatorname{cov}_{Y_{n}}(x)\left|W_{n}\right|\left(\int_{\left(W_{n} \backslash W_{n}^{-}\right) \cap\left(\left(W_{n} \backslash W_{n}^{-}\right)-x\right)} G\left(W_{n}, y\right) G\left(W_{n}, x+y\right) \mathrm{d} y\right) \mathrm{d} x \\
\leq \lim _{n \rightarrow \infty} \frac{c_{1}^{2}\left|W_{n} \backslash W_{n}^{-}\right|}{\left|W_{n}\right|} \int_{\mathbb{R}^{d}} h(x) \mathrm{d} x \\
\leq c \lim _{n \rightarrow \infty} \frac{\left|\partial W_{n} \oplus B_{2 \sqrt{d}}(o)\right|}{\left|W_{n}\right|}=0
\end{aligned}
$$


for some constant $c>0$, where the last equality follows from (2.6). Hence, the second component in the decomposition of $S_{n}^{\prime}$ converges to 0 in mean square. Using Slutsky's theorem, it is sufficient to show that

$$
S_{n}^{*} \stackrel{\mathrm{D}}{\rightarrow} \mathcal{N}\left(0, \sigma^{2}\right) \quad \text { as } n \rightarrow \infty .
$$

Hence, we apply Lemma 4.4 to $S_{n}^{*}=\sum_{z \in U_{n}} Z_{n, z}$, where

$$
Z_{n, z}= \begin{cases}\sqrt{\left|W_{n}\right|} \int_{[z, z+e)}\left(Y_{n}(x)-\mu_{n}\right) G\left(W_{n}, x\right) \mathrm{d} x, & z \in U_{n}, \\ 0, & \text { otherwise. }\end{cases}
$$

It is not difficult to see that the family of random variables $\left\{Z_{n, z}, z \in \mathbb{Z}^{d}\right\}$ given in (4.7) forms an $m_{n}$-dependent random field for any $m_{n} \geq 2\left(a_{n}+D(K)\right)$. By the definition of $Z_{n, z}$, we have $\mathrm{E}\left(Z_{n, z}\right)=0$ for any $z \in U_{n}$. Furthermore, we have

$$
\lim _{n \rightarrow \infty} \mathrm{E}\left(\left(S_{n}^{*}\right)^{2}\right)=\sigma^{2}
$$

by Lemma 4.3 and since, as shown above, $\mathrm{E}\left(\tilde{S}_{n}^{2}\right) \rightarrow 0$. In order to complete the proof, it remains to show that conditions (4.4) and (4.5) are fulfilled. By using Fubini's theorem and (2.9), we obtain

$$
\begin{aligned}
\sum_{z \in U_{n}} \mathrm{E}\left(Z_{n, z}^{2}\right) & \leq \sum_{z \in U_{n}} \frac{c_{1}^{2}}{\left|W_{n}\right|} \int_{[z, z+e)} \int_{[z, z+e)}\left|\operatorname{cov}_{Y_{n}}(x-y)\right| \mathrm{d} x \mathrm{~d} y \\
& \leq \sum_{z \in U_{n}} \frac{c_{1}^{2} \operatorname{var}\left(Y_{n}(o)\right)}{\left|W_{n}\right|} \\
& =c_{1}^{2} \operatorname{var}\left(Y_{n}(o)\right) \frac{\left|W_{n}^{-}\right|}{\left|W_{n}\right|} \\
& \leq c,
\end{aligned}
$$

for all sufficiently large $n$ and some constant $c<\infty$, where the uniform bound provided by the final inequality follows from the facts that $\operatorname{var}\left(Y_{n}(o)\right) \leq h(o)<\infty$ and $\left|W_{n}^{-}\right| /\left|W_{n}\right| \leq 1$ for any $n \geq 1$. Thus, (4.4) holds. Because of the stationarity of $\Xi_{n}$, the random variables

$$
\tilde{Z}_{n, z}=\int_{[z, z+e)} \frac{\left|Y_{n}(x)-\mu_{n}\right|}{\sqrt{\left|W_{n}\right|}} \mathrm{d} x, \quad z \in U_{n},
$$

are identically distributed. By the inequality in (2.9), we also have $\left|Z_{n, z}\right| \leq c_{1} \tilde{Z}_{n, z}$. This yields the following estimates for any $\delta>0$, where $\hat{Z}_{n, o}=\sqrt{\left|W_{n}\right|} \tilde{Z}_{n, o}$ :

$$
\begin{aligned}
m_{n}^{2 d} \sum_{z \in U_{n}} \mathrm{E}\left(Z_{n, z}^{2} \mathbf{1}\left(m_{n}^{2 d}\left|Z_{n, z}\right| \geq \varepsilon\right)\right) & \leq m_{n}^{2 d}\left|W_{n}^{-}\right| c_{1}^{2} \mathrm{E}\left(\tilde{Z}_{n, o}^{2} \mathbf{1}\left(\tilde{Z}_{n, o}^{\delta} \geq \frac{\varepsilon^{\delta}}{c_{1}^{\delta} m_{n}^{2 d \delta}}\right)\right) \\
& \leq \frac{c_{1}^{2+\delta}}{\varepsilon^{\delta}} m_{n}^{2 d(1+\delta)}\left|W_{n}^{-}\right| \mathrm{E}\left(\tilde{Z}_{n, o}^{2+\delta}\right) \\
& \leq \frac{c_{1}^{2+\delta}}{\varepsilon^{\delta}}\left(\frac{m_{n}^{4 d(1+\delta) / \delta}}{\left|W_{n}\right|}\right)^{\delta / 2} \frac{\left|W_{n}^{-}\right|}{\left|W_{n}\right|} \mathrm{E}\left(\hat{Z}_{n, o}^{2+\delta}\right) .
\end{aligned}
$$


Since $m_{n} \geq 2\left(a_{n}+D(K)\right)$, the second factor of the latter expression converges to 0 as $n \rightarrow \infty$ if (4.6) holds for some $\delta>0$. The remaining factors of (4.8) are uniformly bounded in $n$, because $\left|W_{n}^{-}\right| /\left|W_{n}\right| \leq 1$ and

$$
\begin{aligned}
\mathrm{E}\left(\hat{Z}_{n o}^{2+\delta}\right)^{1 /(2+\delta)} & \leq \mathrm{E}\left(\left(\int_{[o, e)}\left(\left|Y_{n}(x)\right|+\left|\mu_{n}\right|\right) \mathrm{d} x\right)^{2+\delta}\right)^{1 /(2+\delta)} \\
& \leq \mathrm{E}\left(\int_{[o, e)}\left|Y_{n}(x)\right|^{2+\delta} \mathrm{d} x\right)^{1 /(2+\delta)}+\left|\mu_{n}\right| \\
& \leq c(K) g_{N(\Xi \cap K)}\left(2^{2+\delta}\right)^{1 /(2+\delta)}+c(K) g_{N(\Xi \cap K)}(2) \\
& <\infty
\end{aligned}
$$

for any $n \geq 1$. Thus, condition (4.5) of Lemma 4.4 is fulfilled.

Notice that condition (4.6) of Lemma 4.5 is satisfied, for example, if the truncation sequence $\left\{a_{n}\right\}$ is given by $a_{n}=r\left(W_{n}\right)^{\eta}$, where $\eta<\delta /(4(1+\delta))$ and $r\left(W_{n}\right)$ denotes the radius of the largest disc that can be inscribed in $W_{n}$.

\subsection{Asymptotic normality of mean-value estimators}

The asymptotic normality proven in Lemma 4.5 for the mean-value estimator associated with the truncated germ-grain model implies an equivalent statement for the original functional.

Theorem 4.1. Let conditions (2.6), (2.8), and (2.9) be fulfilled and assume that

$$
\mathrm{E}\left(\left|M_{0} \oplus \check{K}\right|^{2}\right)<\infty .
$$

Then

$$
\sqrt{\left|W_{n}\right|}\left(\hat{\mu}_{n}-\mu\right) \stackrel{\mathrm{D}}{\rightarrow} \mathcal{N}\left(0, \sigma^{2}\right) \text { as } n \rightarrow \infty,
$$

for $\sigma^{2}=c_{2} \int_{\mathbb{R}^{d}} \operatorname{cov}_{Y}(x) \mathrm{d} x$ with $c_{2}$ as defined in (2.9).

Proof. Under the above assumptions, Lemma 4.5 guarantees that $S_{n}^{\prime} \stackrel{\mathrm{D}}{\rightarrow} \mathcal{N}\left(0, \sigma^{2}\right)$ as $n \rightarrow \infty$, provided that the truncation sequence $\left\{a_{n}\right\}$ satisfies the imposed conditions. Moreover, by setting $S_{n}=\sqrt{\left|W_{n}\right|}\left(\hat{\mu}_{n}-\mu\right)$, we see that the sequence of random variables $S_{n}-S_{n}^{\prime}$ converges to 0 in mean square as $n \rightarrow \infty$ for any truncation sequence $\left\{a_{n}\right\}$ with $\lim _{n \rightarrow \infty} a_{n}=\infty$. This assertion follows directly from Lemmas 2.3, 4.2, and 4.3 together with the fact that $\mathrm{E}\left((Y(o)-\mu)\left(Y_{n}(x)-\mu_{n}\right)\right) \leq h(x)$ for $h(x) \in L^{1}\left(\mathbb{R}^{d}\right)$, as derived in the proof of Lemma 4.1. An application of Slutsky's theorem completes the proof.

By using arguments similar to those in the one-dimensional setting, we can easily extend Theorem 4.1 to the multivariate case. For this, choose sets $K_{i} \in \mathcal{K}$, valuations $f_{i}: \mathcal{R} \rightarrow \mathbb{R}$, and random fields $Y_{i}=\left\{f_{i}\left((\Xi-x) \cap K_{i}\right), x \in \mathbb{R}^{d}\right\}$ for $i=1, \ldots, k$, which are defined on the basis of the same stationary RACS $\Xi$. In addition, let $G_{i}\left(W_{n}, \cdot\right), i=1, \ldots, k$, be weight functions satisfying the conditions

$$
G_{i}\left(W_{n}, x\right)=0 \quad \text { if } x \in \mathbb{R}^{d} \backslash\left(W_{n} \ominus \check{K}_{i}\right), \quad \int_{W_{n}} G_{i}\left(W_{n}, x\right) \mathrm{d} x=1,
$$

$\sup _{y \in W_{n}} G_{i}\left(W_{n}, y\right) \leq \frac{c_{1}}{\left|W_{n}\right|} \quad$ for all $n \geq 1, \quad \lim _{n \rightarrow \infty}\left|W_{n}\right| R_{W_{n}(i j)}(x)=c_{i, j} \quad$ for all $x \in \mathbb{R}^{d}$, 
where $c_{1}, c_{i, j}<\infty$ are some constants and

$$
R_{W_{n}(i j)}(x)=\int_{\mathbb{R}^{d}} G_{i}\left(W_{n}, y\right) G_{j}\left(W_{n}, x+y\right) \mathrm{d} y .
$$

For each $i=1, \ldots, k$, define $\mu_{i}=\mathrm{E}\left(Y_{i}(o)\right)$ and $\hat{\mu}_{n, i}=\int_{W_{n}} Y_{i}(x) G_{i}\left(W_{n}, x\right) \mathrm{d} x$. Then, as in Lemma 2.2, we see that the cross-covariances $\operatorname{cov}\left(\hat{\mu}_{n, i}, \hat{\mu}_{n, j}\right)$ are given by

$$
\operatorname{cov}\left(\hat{\mu}_{n, i}, \hat{\mu}_{n, j}\right)=\int_{\mathbb{R}^{d}} \operatorname{cov}\left(Y_{i}(o), Y_{j}(x)\right) R_{W_{n}(i j)}(x) \mathrm{d} x .
$$

As in Lemmas 2.3 and 4.1, the limits

$$
\sigma_{i, j}=\lim _{n \rightarrow \infty}\left|W_{n}\right| \operatorname{cov}\left(\hat{\mu}_{n, i}, \hat{\mu}_{n, j}\right)
$$

exist and are given by

$$
\sigma_{i, j}=c_{i, j} \int_{\mathbb{R}^{d}} \operatorname{cov}\left(Y_{i}(o), Y_{j}(x)\right) \mathrm{d} x
$$

for any $i, j=1, \ldots, k$, provided that $\mathrm{E}\left(\left|M_{0} \oplus \check{K}_{i}\right|^{2}\right)<\infty$. We are now in a position to formulate a multidimensional analogue of Theorem 4.1.

Theorem 4.2. Let the conditions (2.6), (4.9), and (4.10) be fulfilled and assume that

$$
\mathrm{E}\left(\left|M_{0} \oplus \check{K}_{i}\right|^{2}\right)<\infty \text { for each } i=1, \ldots, k .
$$

Then

$$
\left(\begin{array}{c}
\sqrt{\left|W_{n}\right|}\left(\hat{\mu}_{n, 1}-\mu_{1}\right) \\
\vdots \\
\sqrt{\left|W_{n}\right|}\left(\hat{\mu}_{n, k}-\mu_{k}\right)
\end{array}\right) \stackrel{\mathrm{D}}{\rightarrow} \mathcal{N}_{k}(o, \Sigma), \quad n \rightarrow \infty,
$$

where $\mathcal{N}_{k}(o, \Sigma)$ is a $k$-dimensional Gaussian random vector with zero vector mean and covariance matrix $\Sigma=\left(\sigma_{i, j}\right)$ whose entries are defined by (4.11).

Proof. By the well-known Cramér-Wold device, the assertion is true if and only if, for all $t \in \mathbb{R}^{k} \backslash\{o\}$,

$$
\sqrt{\left|W_{n}\right|} \sum_{i=1}^{k} t_{i}\left(\hat{\mu}_{n, i}-\mu_{i}\right)=\sqrt{\left|W_{n}\right|} \int_{W_{n}} \sum_{i=1}^{k} t_{i}\left(Y_{i}(x)-\mu_{i}\right) G_{i}\left(W_{n}, x\right) \mathrm{d} x \stackrel{\mathrm{D}}{\rightarrow} \mathcal{N}\left(0, \sigma^{2}\right),
$$

where $\sigma^{2}=t^{\top} \Sigma t$. The above convergence can be proven analogously to Theorem 4.1.

\section{Asymptotic normality for $\beta$-mixing random measures}

In the previous section, we considered germ-grain models driven by a Poisson point process. Now we show how the above results can be extended to a more general setting in which we do not assume that the point process $\left\{X_{i}\right\}$ of germs is necessarily Poisson, but rather that it satisfies some mixing condition.

Let us begin by recalling some basic notions from mixing; see, e.g. [2] for further details. Consider the probability space $(\Omega, \mathcal{A}, \mathrm{P})$ and let $\mathcal{A}_{1}, \mathcal{A}_{2} \subset \mathcal{A}$ be two $\sigma$-subalgebras of $\mathcal{A}$. The $\beta$-mixing coefficient (also called the absolute regularity coefficient) of $\mathcal{A}_{1}$ and $\mathcal{A}_{2}$ is defined by

$$
\beta\left(\mathcal{A}_{1}, \mathcal{A}_{2}\right)=\frac{1}{2} \sup \sum_{k} \sum_{\ell}\left|\mathrm{P}\left(A_{k} \cap B_{\ell}\right)-\mathrm{P}\left(A_{k}\right) \mathrm{P}\left(B_{\ell}\right)\right|,
$$


where the supremum is taken over all pairs of finite partitions $\left\{A_{k}\right\}$ and $\left\{B_{\ell}\right\}$ of $\Omega$ with $A_{k} \in \mathcal{A}_{1}$ for all $k$ and $B_{\ell} \in \mathcal{A}_{2}$ for all $\ell$. Furthermore, for any pair of bounded Borel sets $C_{1}, C_{2} \in$ $\mathcal{B}_{0}\left(\mathbb{R}^{d}\right)$, let $\rho\left(C_{1}, C_{2}\right)=\inf \left\{\left|x_{1}-x_{2}\right|: x_{1} \in C_{1}, x_{2} \in C_{2}\right\}$ denote the distance between $C_{1}$ and $C_{2}$. For any $s>0$, the $\beta$-mixing rate $\beta_{X}(s)$ of a point process $X=\left\{X_{i}\right\}$ in $\mathbb{R}^{d}$ is defined by

$$
\beta_{X}(s)=\sup \left\{\beta\left(\sigma\left(N_{X}\left(C_{1}\right)\right), \sigma\left(N_{X}\left(C_{2}\right)\right)\right): C_{1}, C_{2} \in \mathcal{B}_{0}\left(\mathbb{R}^{d}\right), \rho\left(C_{1}, C_{2}\right) \geq s\right\},
$$

where $\sigma\left(N_{X}(C)\right)$ is the $\sigma$-algebra generated by $N_{X}(C)=\operatorname{card}\left\{i: X_{i} \in C\right\}$.

Let $\Xi$ be a germ-grain model of the form (2.1) for some stationary point process $X=$ $\left\{X_{i}\right\}$ and convex grains, and let the random field $Y=\left\{Y(x), x \in \mathbb{R}^{d}\right\}$ be given by (2.5). If $X$ is a Poisson process then $\beta_{X}(s)=0$ for all $s>0$, and Theorem 4.1 implies that $\sqrt{\left|W_{n}\right|}\left(\hat{\mu}_{n}-\mu\right)$ converges weakly to a Gaussian random variable if $\mathrm{E}\left(\left|M_{0} \oplus \check{K}\right|^{2}\right)<\infty$. Using similar arguments, we can even consider a slightly more general case, in which $\beta_{X}(s)=0$ for all $s$ greater than or equal to some $s_{0}>0$, which holds, for example, if $X$ is a Matérn cluster process; see, e.g. [15, p. 159] for a definition. Then, the assertions of Lemmas 4.2 and 4.3 hold if $g_{N(\Xi \cap K)}(4)<\infty$ and if there exists an integrable function $h: \mathbb{R}^{d} \rightarrow \mathbb{R}_{+}$such that $\left|\operatorname{cov}_{Y_{n}}(x)\right| \leq h(x)$ for any $x \in \mathbb{R}^{d}$ and $n \geq n_{0}$, where $n_{0} \geq 0$ is some integer. Moreover, Theorem 4.1 remains valid, with the range of dependency, $m_{n} \geq 2\left(a_{n}+D(K)\right)$, in the proof of Lemma 4.5 replaced by $m_{n}^{\prime} \geq 2\left(a_{n}+D(K)\right)+s_{0}$.

In the remaining part of this section, we briefly discuss a different technique of proving that the normal convergence (4.1) holds. (Notice that this technique has been used in [7] for another general class of functionals of germ-grain models.) Let $\left\{Z_{z}, z \in \mathbb{Z}^{d}\right\}$ be a stationary random field and let $\left\{U_{n}, n \geq 1\right\}$ be a sequence of finite subsets of $\mathbb{Z}^{d}$ with $\lim _{n \rightarrow \infty} \operatorname{card}\left(U_{n}\right)=\infty$. Assuming that there exist functions $b_{Z}^{*}$ and $b_{Z}^{* *}$ on $[0, \infty)$ such that

$$
\beta\left(\sigma\left(Z_{z},|z|<p+1\right), \sigma\left(Z_{z},|z| \geq p+q\right)\right) \leq \begin{cases}b_{Z}^{*}(q), & q>p=0 \\ p^{d-1} b_{Z}^{* *}(q), & p \geq q \geq 1,\end{cases}
$$

the following central limit theorem for absolutely regular random fields holds; see Theorem 6.1 of [6].

Lemma 5.1. Let $\left\{Z_{z}, z \in \mathbb{Z}^{d}\right\}$ satisfy the following conditions:

$$
\begin{gathered}
\mathrm{E}\left(Z_{0}\right)=0, \quad \mathrm{E}\left(\left|Z_{0}\right|^{2+\delta}\right)<\infty \quad \text { for some } \delta>0, \\
\sum_{q \geq 1} q^{d-1}\left(b_{Z}^{*}(q)\right)^{\delta /(2+\delta)}<\infty, \quad \lim _{q \rightarrow \infty} q^{2 d-1} b_{Z}^{* *}(q)=0 .
\end{gathered}
$$

Then $\operatorname{card}\left(U_{n}\right)^{-1 / 2} S_{n} \stackrel{\mathrm{D}}{\rightarrow} \mathcal{N}\left(0, \sigma^{2}\right)$, where $S_{n}=\sum_{z \in U_{n}} Z_{z}$ and $\sigma^{2}=\sum_{z \in \mathbb{Z}^{d}} \mathrm{E}\left(Z_{0} Z_{z}\right)$ is absolutely convergent.

Now let $W_{n}$ be the $d$-dimensional cube $[-n, n)^{d}$, set $W_{n}^{K}=\left[-n+D^{\prime}(K), n-D^{\prime}(K)\right)^{d}$, where $D^{\prime}(K)$ is the smallest integer greater than the norm $D(K)$, and define $G\left(W_{n}, x\right)=$ $\mathbf{1}\left(x \in W_{n}^{K}\right) /\left|W_{n}^{K}\right|$ for any $x \in \mathbb{R}^{d}$ and all $n$ large enough that $\left|W_{n}^{K}\right|>0$. Furthermore, let $\Xi=\bigcup_{i=1}^{\infty}\left(M_{i}+X_{i}\right)$ be a germ-grain model in which the stationary point process $X=\left\{X_{i}\right\}$ has the following mixing property. As in [7], we assume that there exists a nonincreasing function $b_{X}(\cdot)$ on $[1, \infty)$ such that, for all $a, \Delta \geq 1$,

$$
\beta\left(\sigma\left(N_{X}\left([-a, a]^{d}\right)\right), \sigma\left(N_{X}\left(\mathbb{R}^{d} \backslash[-a-\Delta, a+\Delta]^{d}\right)\right)\right) \leq b_{X}(\Delta)\left(\frac{a}{\min \{a, \Delta\}}\right)^{d-1} .
$$


Theorem 5.1. Let $\Xi$ be a stationary germ-grain model satisfying (5.2) with non-empty typical grain $M_{0} \in \mathcal{R}$. If there exist some $\delta, \varepsilon>0$ such that

$$
\mathrm{E}\left(|Y(o)|^{2+\delta}\right)<\infty, \quad \mathrm{E}\left(D^{2 d(\delta+1) / \delta+\varepsilon}\left(M_{0}\right)\right)<\infty,
$$

and

$$
\sum_{n=1}^{\infty} n^{d-1} b_{X}(n)^{\delta /(2+\delta)}<\infty
$$

then

$$
\sqrt{\left|W_{n}\right|} \int_{W_{n}}(Y(x)-\mu) G\left(W_{n}, x\right) \mathrm{d} x \stackrel{\mathrm{D}}{\rightarrow} \mathcal{N}\left(0, \sigma^{2}\right), \quad n \rightarrow \infty,
$$

where $\sigma^{2}=\int_{\mathbb{R}^{d}} \operatorname{cov}_{Y}(x) \mathrm{d} x$.

The proof of Theorem 5.1 is similar to that of Theorem 6.2 of [7]. Hence, we merely sketch the main steps. We consider the set function $\eta: \mathscr{B}_{0}\left(\mathbb{R}^{d}\right) \rightarrow \mathbb{R}$ with

$$
\eta(B)=\int_{B}(Y(x)-\mu) \mathrm{d} x, \quad B \in \mathcal{B}_{0}\left(\mathbb{R}^{d}\right),
$$

and show that $\left|W_{n}^{K}\right|^{-1 / 2} \eta\left(W_{n}^{K}\right) \stackrel{\mathrm{D}}{\rightarrow} \mathcal{N}\left(0, \sigma^{2}\right)$ as $n \rightarrow \infty$, using Lemma 5.1. That is, the latter expression can be written as

$$
\left|W_{n}^{K}\right|^{-1 / 2} \eta\left(W_{n}^{K}\right)=\operatorname{card}\left(U_{n}\right)^{-1 / 2} \sum_{z \in U_{n}} Z_{z}, \quad U_{n}=\left\{z \in \mathbb{Z}^{d}:[z, z+e) \subseteq W_{n}^{K}\right\},
$$

for the stationary random field $Z=\left\{Z_{z}, z \in \mathbb{Z}^{d}\right\}$ with $Z_{z}=\int_{[z, z+e)}(Y(x)-\mu) \mathrm{d} x$. Then we have $\mathrm{E}\left(Z_{0}\right)=0$ and $\mathrm{E}\left(\left|Z_{0}\right|^{2+\delta}\right) \leq \mathrm{E}\left(|Y(0)-\mu|^{2+\delta}\right)$, where the latter bound is finite by assumption (5.3), and, finally,

$$
\sigma^{2}=\sum_{z \in \mathbb{Z}^{d}} \mathrm{E}\left(Z_{0} Z_{z}\right)=\sum_{z \in \mathbb{Z}^{d}} \int_{[o, e)} \int_{[z, z+e)} \operatorname{cov}_{Y}(x-y) \mathrm{d} x \mathrm{~d} y=\int_{\mathbb{R}^{d}} \operatorname{cov}_{Y}(x) \mathrm{d} x .
$$

To check the conditions in (5.1), let $\sigma_{\eta}(B)$ be the $\sigma$-algebra generated by $\left\{\eta\left(B^{\prime}\right), B^{\prime} \subseteq B, B^{\prime} \in\right.$ $\left.\mathcal{B}_{0}\left(\mathbb{R}^{d}\right)\right\}$. For any $p, q \in \mathbb{N}$, there exist $a, \Delta \geq 0$ satisfying $\beta\left(\sigma\left(Z_{z},|z|<p+1\right), \sigma\left(Z_{z},|z| \geq p+q\right)\right) \leq \beta\left(\sigma_{\eta}\left([-a, a)^{d}\right), \sigma_{\eta}\left(\mathbb{R}^{d} \backslash[-a-\Delta, a+\Delta)^{d}\right)\right)$.

The right-hand side of the last estimate can be bounded by the $\beta$-mixing coefficient of the underlying point process $X$ and certain moments of $D\left(M_{0}\right)$. This can be seen by following the proofs of Lemmas 5.1 and 5.2 of [7], whence

$$
\begin{aligned}
& \beta\left(\sigma_{\eta}\left([-a, a)^{d}\right), \sigma_{\eta}\left(\mathbb{R}^{d} \backslash[-a-\Delta, a+\Delta)^{d}\right)\right) \\
& \leq \beta\left(\sigma\left(N_{X}\left(\left[-a-\frac{\Delta}{4}, a+\frac{\Delta}{4}\right)^{d}\right)\right), \sigma\left(N_{X}\left(\mathbb{R}^{d} \backslash\left[-a-\frac{3 \Delta}{4}, a+\frac{3 \Delta}{4}\right)^{d}\right)\right)\right) \\
& \quad+\lambda d 2^{d+1}\left(\left(\frac{\Delta+4 a}{\Delta}\right)^{d+1}+\left(\frac{\Delta+12 a}{\Delta}\right)^{d+1}\right) \\
& \quad \times \mathrm{E}\left(D^{d}\left(M_{0} \oplus \check{K}\right) \mathbf{1}\left(D\left(M_{0} \oplus \check{K}\right)>\frac{\Delta}{4}\right)\right) \\
& \leq\left(\frac{a}{\min \{a, \Delta\}}\right)^{d-1}\left(c_{1}(d) b_{X}\left(\frac{\Delta}{2}\right)+c_{2}(d) \mathrm{E}\left(D^{d}\left(M_{0} \oplus \check{K}\right) \mathbf{1}\left(D\left(M_{0} \oplus \check{K}\right)>\frac{\Delta}{4}\right)\right)\right)
\end{aligned}
$$


for some finite constants $c_{1}(d)$ and $c_{2}(d)$, employing assumption (5.2) and the fact that $\mathrm{E}\left(D^{d}\left(M_{0} \oplus \check{K}\right)\right)<\infty$. In the proofs of the above-mentioned lemmas, let

$$
\Xi_{B}=\bigcup_{X_{i} \in B}\left(M_{i}+X_{i}\right)
$$

denote the germ-grain model restricted to germs within $B \in \mathscr{B}\left(\mathbb{R}^{d}\right)$, define

$$
Y_{B}(x)=f\left(\left(\Xi_{B}-x\right) \cap K\right),
$$

and let $\eta_{B}\left(B^{\prime}\right)=\int_{B^{\prime}} Y_{B}(x) \mathrm{d} x$ for any $B^{\prime} \in \mathscr{B}_{0}\left(\mathbb{R}^{d}\right)$. Next, define

$$
b_{\eta}(\Delta)=c_{1}(d) b_{X}\left(\frac{1}{2} \Delta\right)+c_{2}(d) \mathrm{E}\left(D^{d}\left(M_{0} \oplus \check{K}\right) \mathbf{1}\left(D\left(M_{0} \oplus \check{K}\right)>\frac{1}{4} \Delta\right)\right)
$$

and let $b_{Z}^{*}(\Delta)=b_{\eta}(\Delta)$ and $b_{Z}^{* *}(\Delta)=b_{\eta}(\Delta) / \Delta^{d-1}$. Hence, the proof of Theorem 5.1 is completed by noting that (5.3) and (5.4) imply

$$
\begin{aligned}
\lim _{\Delta \rightarrow \infty} & \Delta^{d} b_{Z}^{* *}(\Delta) \\
\leq & c_{1}(d) \lim _{\Delta \rightarrow \infty} \mathrm{E}\left(D\left(M_{0} \oplus \check{K}\right) \mathbf{1}\left(D\left(M_{0} \oplus \check{K}\right)>\Delta\right)\right) \lim _{\Delta \rightarrow \infty} \Delta^{d-1} b_{X}^{\delta /(2+\delta)}\left(\frac{1}{2} \Delta\right) \\
& +4^{d} c_{2}(d) \lim _{\Delta \rightarrow \infty} \mathrm{E}\left(D^{2 d}\left(M_{0} \oplus \check{K}\right) \mathbf{1}\left(D\left(M_{0} \oplus \check{K}\right)>\frac{1}{4} \Delta\right)\right)=0
\end{aligned}
$$

and that there exists some $\Delta_{0}$ such that

$$
\begin{aligned}
\sum_{\Delta \geq \Delta_{0}} & \Delta^{d-1}\left(b_{Z}^{*}(\Delta)\right)^{\delta /(2+\delta)} \\
\leq & 2^{d-1} c_{1}^{\delta /(2+\delta)}(d) \sum_{\Delta \geq 1} \Delta^{d-1}\left(b_{X}(\Delta)\right)^{\delta /(2+\delta)} \\
& +4^{d-1} c_{2}^{\delta /(2+\delta)}(d) \sum_{\Delta \geq 1} \Delta^{d-1} \mathrm{E}\left(D^{d}\left(M_{0} \oplus \check{K}\right) \mathbf{1}\left(D\left(M_{0} \oplus \check{K}\right)>\Delta\right)\right)^{\delta /(2+\delta)} \\
\leq & \tilde{c}_{1}(d)+\tilde{c}_{2}(d) \mathrm{E}\left(D^{2 d(\delta+1) / \delta+\varepsilon}\left(M_{0} \oplus \check{K}\right)\right) \sum_{\Delta \geq 1} \Delta^{-\left(1+\varepsilon^{\prime}\right)}<\infty
\end{aligned}
$$

with

$$
\begin{aligned}
& \tilde{c}_{1}(d)=2^{d-1} c_{1}^{\delta /(2+\delta)}(d) \sum_{\Delta \geq 1} \Delta^{d-1}\left(b_{X}(\Delta)\right)^{\delta /(2+\delta)}<\infty, \\
& \tilde{c}_{2}(d)=4^{d-1} c_{2}^{\delta /(2+\delta)}(d)<\infty .
\end{aligned}
$$

For the Boolean model $\Xi=\bigcup_{i=1}^{\infty}\left(M_{i}+X_{i}\right)$, with $M_{0} \in \mathcal{R} \backslash\{\varnothing\}$ such that the minimal number of convex components of $M_{0}$ is bounded by some finite constant, the conditions of Theorem 5.1 are fulfilled if $\mathrm{E}\left(D^{2 d(\delta+1) / \delta+\varepsilon}\left(M_{0}\right)\right)<\infty$ holds for some $\delta, \varepsilon>0$. Notice that this integrability condition is stronger than the assumption $\mathrm{E}\left(\left|M_{0} \oplus \breve{K}\right|^{2}\right)$ made in Theorems 4.1 and 4.2. Further examples of point processes $X$ satisfying conditions (5.2) and (5.4) can be found in [7]. We also remark that in the special case $Y=Z_{r}$ considered in (3.2) with arbitrary $X$ and $M_{0} \in \mathcal{K}^{\prime}$, the integrability condition $\mathrm{E}\left(N^{(2+\delta) d}\left(\Xi \cap B_{1}(o)\right)\right)<\infty$ implies that $\mathrm{E}\left(|Y(x)|^{2+\delta}\right)<\infty$ for $\delta>0$, provided that there is almost surely no boundary point of $\Xi$ where more than $d$ germs overlap; see Section 4 of [11]. 


\section{Acknowledgements}

We would like to thank Jan Rataj and the anonymous referees for their useful comments, which helped us to improve the manuscript.

\section{References}

[1] Böhm, S., Heinrich, L. AND Schmidt, V. (2004). Asymptotic properties of estimators for the volume fraction of jointly stationary random sets. Statist. Neerlandica $\mathbf{5 8 , 3 8 8 - 4 0 6 .}$

[2] Doukhan, P. (1994). Mixing: Properties and Examples (Lecture Notes Statist. 85). Springer, New York.

[3] Hall, P. (1988). Introduction to the Theory of Coverage Processes. John Wiley, New York.

[4] Heinrich, L. (1988). Asymptotic behaviour of an empirical nearest-neighbour distance function for stationary Poisson cluster processes. Math. Nachr. 136, 131-148.

[5] Heinrich, L. (1993). Asymptotic properties of minimum contrast estimators for parameters of Boolean models. Metrika 31, 349-360.

[6] Heinrich, L. (1994). Normal approximations for some mean-value estimates of absolutely regular tessellations. Math. Meth. Statist. 3, 1-24.

[7] Heinrich, L. ANd Molchanov, I. (1999). Central limit theorem for a class of random measures associated with germ-grain models. Adv. Appl. Prob. 31, 283-314.

[8] Ivanov, A. V. And LeOnenKo, N. N. (1989). Statistical Analysis of Random Fields. Kluwer, Dordrecht.

[9] MASE, S. (1982). Asymptotic properties of stereological estimators of volume fraction for stationary random sets. J. Appl. Prob. 19, 111-126.

[10] Molchanov, I. S. (1997). Statistics of the Boolean Model for Practitioners and Mathematicians. John Wiley, Chichester.

[11] Schmidt, V. AND Spodarev, E. (2005). Joint estimators for the specific intrinsic volumes of stationary random sets. Stoch. Process. Appl. 115, 959-981.

[12] Schneider, R. (1993). Convex Bodies: The Brunn-Minkowski Theory (Encyclopedia Math. Appl. 44). Cambridge University Press.

[13] SchneIder, R. AND WeIL, W. (2000). Stochastische Geometrie. Teubner, Stuttgart.

[14] Spodarev, E. AND Schmidt, V. (2005). On the local connectivity number of stationary random closed sets. In Proc. 7th Internat. Symp. Math. Morphology, eds C. Ronse et al., Kluwer, Dordrecht, pp. 343-356.

[15] Stoyan, D., Kendall, W. S. and Mecke, J. (1995). Stochastic Geometry and its Applications, 2nd edn. John Wiley, Chichester. 